\title{
Etude des effets de pression sur la dynamique de relaxation du krypton après excitation sélective par un laser ultraviolet à $125 \mathrm{~nm}$
}

\author{
A. Kanaev, V. Zafiropulos*, M. Aït-Kaci**, L. Museur**, H. Nkwawo** et M. C. Castex**
}

Institut Lebedev, Academie des Sciences, Moscou, Russie

* FORTH, Heraklion, Grece

** Laboratoire de Physique des Lasers, Av. J.B. Clément, 93430 Villetaneuse, France

Abstract : New approach for the understanding of the energy relaxation dynamics of excited atoms involving a long - lived molecular " reservoir " is presented here in the case of krypton. The one photon selective excitation is realized with a coherent VUV light source generated via nonlinear processes in mercury vapor.

Une nouvelle approche pour la compréhension des processus cinétiques associés au déclin d'atomes métastables $5 \mathrm{~s}(3 / 2)^{0}{ }_{2}\left({ }^{3} \mathrm{P}_{2}\right)$ de Krypton est présentée ici à partir de l'analyse temporelle du signal de fluorescence du $\mathrm{Kr}_{2}{ }^{*}$ ( $2 \mathrm{e}$ continuum).

L'excitation sélective à $125.1 \mathrm{~nm}$ est réalisée grâce à un laser VUV d'une extrême simplicité mis au point au laboratoire (1). Le rayonnement cohérent VUV crée à 125.1 nm (Fig. 1), intense ( $10^{11} \mathrm{ph} /$ pulse ), pulsé ( $20 \mathrm{~Hz}$ ), polarisé, de finesse spectrale $7.10^{-4} \mathrm{~nm}$, est obtenu par mélange de fréquences à 4 ondes $\left(2 \omega_{1}+\omega_{2}\right)$ dans la vapeur de mercure (Fig. 2).

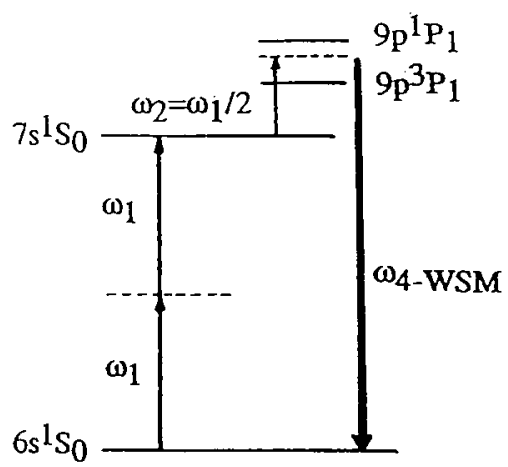

Fig. 2 : Diagramme d'énergie du $\mathrm{Hg}$

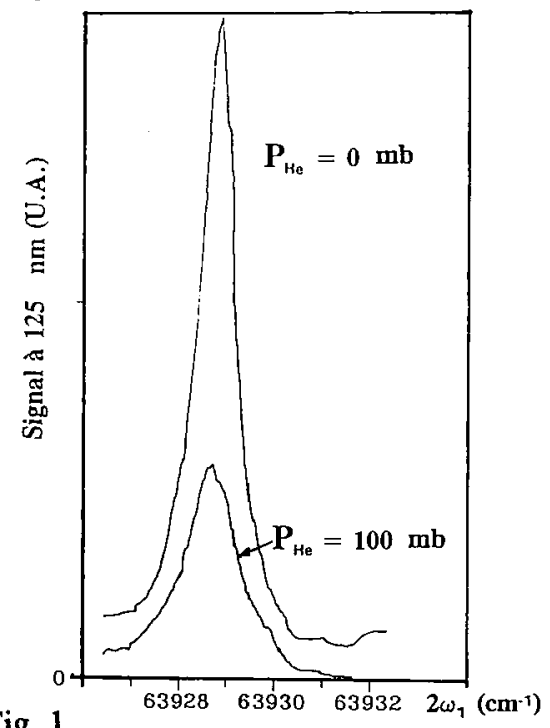

Fig. 1

Ce milieu non linéaire offre la possibilité unique d'utiliser une combinaison de fréquences $\omega_{2}=\omega_{1} / 2$ telles que :

- le rendement de conversion $\left(5.10^{-4}\right)$ du processus non linéaire peut être amplifié par un effet de double résonance,

- le dispositif laser ne nécessite qu'un colorant doublé pompé par un Yag doublé,

- la cuve au mercure utilisée à $\mathrm{T}$ ambiante $\left(\mathrm{P}_{\mathrm{Hg}} \cong 10^{-3}\right.$ torr), ne nécessite pas l'utilisation d'un gaz tampon (He), qui aurait pour effet à cette $\lambda$ de diminuer l'intensité du signal VUV, (Fig. 3). 


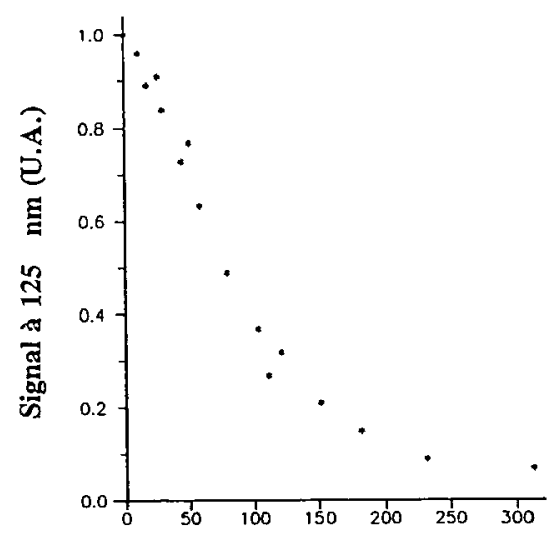

Fig. 3 Pression en mbar $\mathbf{P}_{\mathrm{He}}$

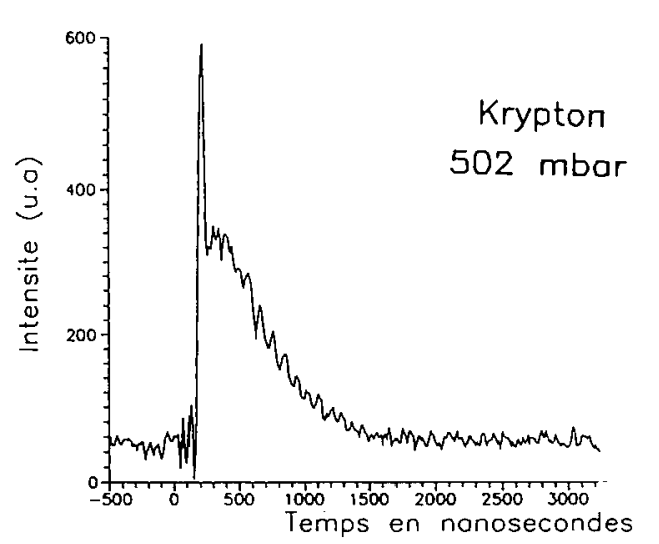

Fig. 4

L'intensité du rayonnement VUV ainsi crée à $125.1 \mathrm{~nm}$ permet d'exciter sélectivement le niveau métastable $\mathrm{Kr}\left({ }^{3} \mathrm{P}_{2}\right)\left(\mathrm{E}_{\mathrm{at}}-\mathrm{E}_{\mathrm{ex}} \ll \mathrm{KT}\right)$. Nous nous sommes principalement intéressés aux temps de déclin (composante lente) mesurés à des pressions de $\mathrm{Kr}$ comprises entre 0.2 et 0.5 bar (Fig. 4). Dans ce domaine de pressions, on observe une dépendance linéaire de $1 / \tau$ avec $P$ donnant, par extrapolation à pression nulle, une valeur $1 / \tau_{0}$ négative (Fig. 5). Nous montrons que ces résultats s'expliquent par le peuplement collisionnel d'un réservoir moléculaire métastable $1 \mathrm{~g}$ associé au niveau $\mathrm{Kr}^{*}\left({ }^{3} \mathrm{P}_{2}\right)$, les échanges d'énergie $\mathrm{Kr}^{*}\left({ }^{3} \mathrm{P}_{2}\right)+2 \mathrm{Kr} \leftarrow \rightarrow \mathrm{Kr}_{2}{ }^{*}(1 \mathrm{~g})+\mathrm{Kr}$ contrôlant le processus de formation de l'excimère $\mathrm{Kr}_{2}{ }^{*}$ ( $1 \mathrm{u}$ ).

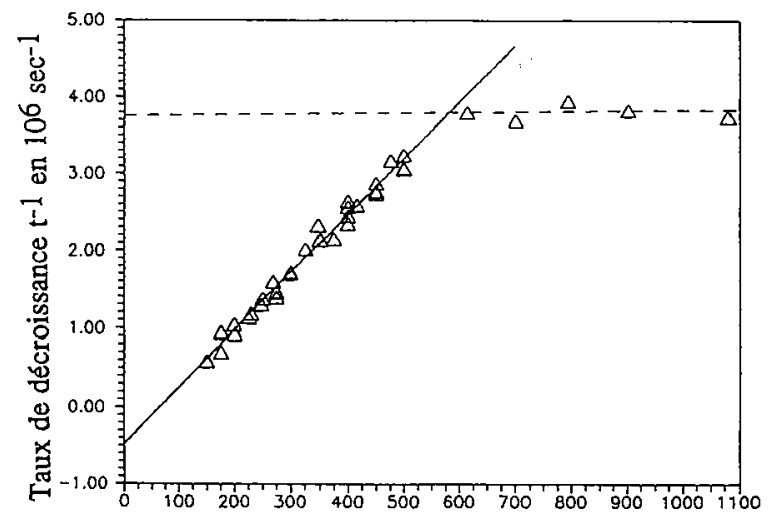

Fig. 5

Pression de Krypton en mbar

Aux pressions $P>0.5$ bar, le temps de déclin de la composante lente est attribuée au temps de déclin radiatif de l'état $1 \mathrm{lu}$. La valeur ( $265 \mathrm{~ns})$ que nous obtenons par extrapolation à pression nulle est en excellent accord avec celles d'autres auteurs (2-4).

(1)- H. Nkwawo, C. Maĩnos, M. Aitt-Kaci, L. Museur and M. C. Castex, J. Phys.C7. 481 (91).

(2)- E. Audouard, P. Laporte, J. L. Subtil and N. Damany, J. Chem, Phys. 89. 6176 (88).

(3)- Y. Salamero, A. Birot, H. Brunet, J. Galy, P. Millet and J. P. Montagne, J. Phys. B. 12.419 (79).

(4)- J. E. Velazco, J. H. Kolts and D. W. Setser, J. Chem, Phys. 69. 4357 (78). 